\title{
Preconception counseling, fertility, and pregnancy complications after abdominal organ transplantation: a survey and cohort study of 532 recipients
}

\begin{abstract}
Rupley DM, Janda AM, Kapeles SR, Wilson TM, Berman D, Mathur AK. Preconception counseling, fertility, and pregnancy complications after abdominal organ transplantation: a survey and cohort study of 532 recipients.
\end{abstract}

Abstract: Background: Pregnancy after solid organ transplant is a significant priority for transplant recipients but how patients report being counseled is unknown.

Methods: We performed a single-center retrospective cohort study and telephone survey of female patients ages 18-49 at the time of kidney, pancreas, or liver transplant from 2000 to $2012(\mathrm{n}=532)$. Data on pregnancy counseling, fertility, and maternal, fetal- and transplantspecific outcomes were collected. Multivariate Cox models assessed the impact of pregnancy on graft-specific outcomes.

Results: The survey response rate was $29 \%(\mathrm{n}=152)$. One-third $(\mathrm{n}=51)$ of women were actively counseled against pregnancy by one or more providers. A total of 17 pregnancies occurred among nine patients $(5.9 \%$ ), with $47 \%$ live births, $47 \%$ early embryonic demises, $5.9 \%$ stillbirths. Of live births, $50 \%$ were premature. Gestational complications, including diabetes, hypertension, and preeclampsia were present in $88 \%$ of mothers. Pregnancy after transplant was associated with higher rates of acute rejection than nulliparous transplant recipients ( $33 \%$ vs. $5.6 \%, p=0.07$ ) but did not significantly affect graft survival $(\mathrm{HR}=1.00,95 \% \mathrm{CI} 0.99-1.01)$, after stratifying by organ and adjusting for clinical factors.

Conclusion: This study suggests that transplant patients are being counseled against pregnancy despite acceptable risks of complications and no specific effects on long-term graft function.

\author{
Devon M. Rupleya, Allison M. \\ Janda ${ }^{a}$, Steven R. Kapeles ${ }^{a}$, Tim \\ M. Wilson ${ }^{a}$, Deborah Berman ${ }^{b}$ and \\ Amit K. Mathur ${ }^{\mathrm{c}}$ \\ aSection of Transplantation Surgery, \\ Department of Surgery, University of Michigan, \\ ${ }^{b}$ Department of Obstetrics and Gynecology, \\ University of Michigan, Ann Arbor, MI and \\ cDivision of Transplant Surgery, Department of \\ Surgery, Mayo Clinic, Phoenix, AZ, USA
}

Key words: abdominal organ - acute rejection after pregnancy - fertility in transplant patients - fetal outcomes - graft failure after pregnancy - preconception counseling pregnancy after transplant - pregnancy outcomes - prenatal counseling - transplant

Corresponding author: Amit K. Mathur, MD, MS, Division of Transplant Surgery, Department of Surgery, Mayo Clinic, 5777 East Mayo Blvd., Phoenix, AZ 85054, USA.

e-mail: mathur.amit@mayo.edu

Conflict of interest: None.

Accepted for publication 18 May 2014
As the size of the reproductive-aged transplant population continues to grow, expectations regarding the feasibility, safety, and advisability of pregnancy in solid organ recipients have changed substantially. Since 1958, over 14000 births have been reported worldwide in transplant recipients of solid organs (1). Despite the increasing prevalence of pregnancy in this population, extensive gaps in understanding of the risks to the graft, the organ recipient, and the fetus exist among patients and their providers. These gaps deter effective counseling regarding fertility, pregnancy, and the implications of these events on graft function after solid organ transplant.
Currently, counseling patients about pregnancy after transplant is hampered by the lack of highquality clinical data. Registries of pregnancy outcomes in solid organ transplant recipients have shown that pregnancy is feasible with a higher gestational complication rate compared to normal controls, and without a demonstrable effect on long-term graft function $(1,2)$. However, these data are not systematically collected and are voluntarily provided by patients and centers (3). These studies have been criticized for inflating live birthrates reported in the post-transplant population by almost $10 \%$ over normal controls (4-7). Further information regarding the consequences of 
immunosuppression on pregnancy outcomes are also needed, but this fund of knowledge is limited by small studies with heterogeneous drug regimens. Although most pregnant solid organ recipients are managed as high-risk obstetrical patients, standard management procedures or protocols have yet to be implemented for managing this population on a wide scale. Although one study by McKay et al. in 2006 (8) was performed on patient counseling as reported by providers, there are few data from transplant patients regarding their counseling experience focusing on pregnancy following transplantation. This study reported $82 \%$ of providers advise against pregnancy following transplantation, with $30 \%$ of these providers basing their recommendation on institutional policies or protocols (8). The lack of standardized and widely implemented recommendations for transplant patients regarding pregnancy is a significant problem that may affect clinical decision-making and quality of life. A better understanding of how patients are counseled on these risks during the transplant process is warranted.

In this analysis, we conducted a telephone survey and retrospective cohort study on 532 female solid organ transplant recipients at our center to assess how patients have been counseled about fertility and pregnancy, as well as determine predictors of variation in maternal and fetal outcomes, and delineate the effect of pregnancy on transplant outcomes.

\section{Methods}

\section{Survey design and execution}

The study was approved by the University of Michigan Institutional Review Board. The study population was derived as shown in Fig. 1. A query was run through the Organ Transplant Information System (OTIS), the medical records system used at the University of Michigan for every transplant patient, to obtain the contact information for females who underwent solid organ transplantation for a kidney, liver, or pancreas between the ages of 18-49 from 2000 to 2012 at the University of Michigan Health System. Patients meeting survey criteria $(\mathrm{n}=532)$ were contacted via telephone and asked to voluntarily participate in the 10-25 min survey (Appendix 1). The surveys were conducted by trained personnel under direction of the study authors and the principal investigator (DR, AJ, AM). Interviewers attempted to contact each patient on three separate occasions. Patients were not included in further analysis if contact was not established. Collected

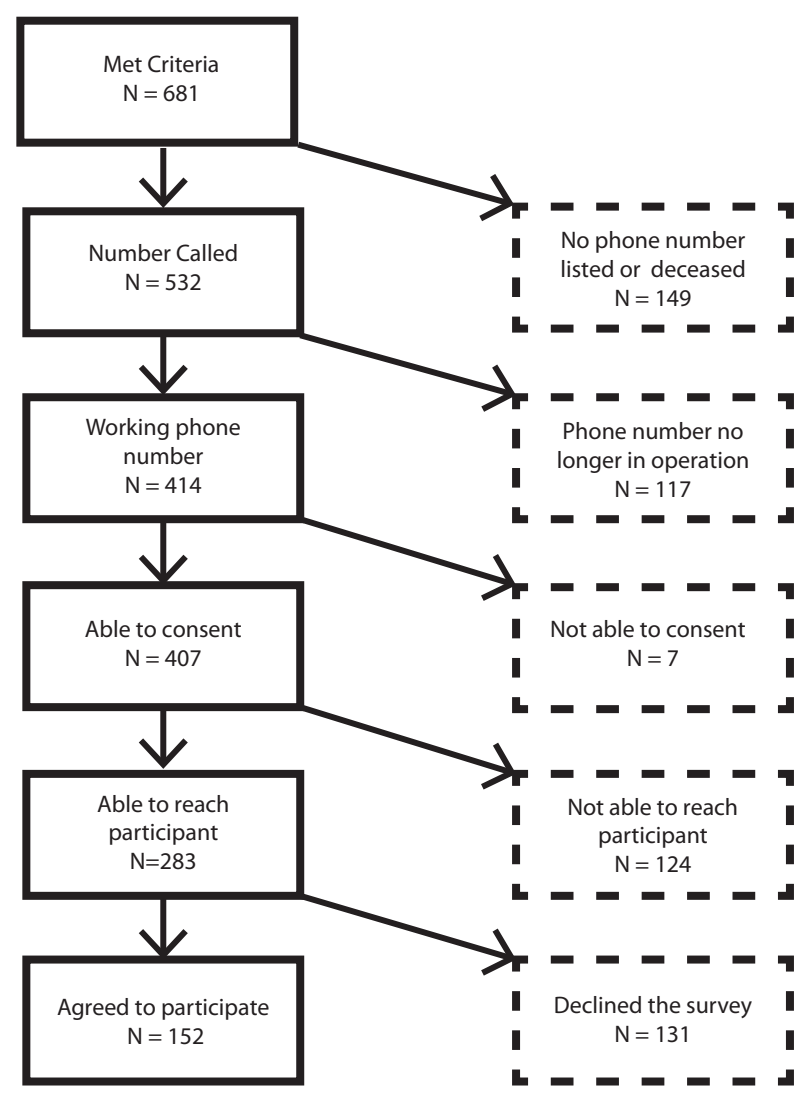

Fig. 1. Calling tree flow chart. Patients included in the study are shown in solid boxes. A total of 152 patients participated in the survey.

data included information regarding patient demographics, socioeconomic status, clinical factors, and graft outcomes. Subjects were not compensated or rewarded for participation.

All collected information was de-identified and stored on a secure server. These data were then analyzed in terms of counseling received, graft function post-pregnancy, maternal gestational complications, delivery complications, and neonatal outcomes. Patients with prior history of permanent sterilization including previous hysterectomy, tubal ligation, or Essure ${ }^{\circledR}$ (Conceptus Inc, Mountain View, CA, USA) were excluded. Data collection on prenatal counseling included whether counseling against pregnancy was given at the time of transplantation, and which provider performed the counseling. Variables of interest to assess maternal outcomes included: rate of conception, frequencies of live birth, early embryonic loss, voluntary interruption of pregnancy, stillbirth, and ectopic pregnancy. Additionally, rates of gestational diabetes, gestational hypertension, preeclampsia, delivery complications, and frequency of breastfeeding were recorded. Neonatal outcome variables included: gestational age at delivery, birth weight, reported neonatal complications, and ongoing childhood- 
Prenatal counseling, pregnancy, and transplant outcomes

chronic medical conditions. Transplant outcomes related to graft function included: self-reported acute rejection episodes, immunosuppressive medications, self-reported graft failure defined as return to dialysis or death for kidney transplant, re-transplant or death for liver transplant, and documented return to insulin usage for pancreas transplant. The data obtained via the survey regarding graft outcomes such as rejection episodes and graft failure were verified with a query of OTIS and manual chart review.

\section{Retrospective cohort study design}

To verify survey responses, chart reviews of the study cohort were performed to gather specific clinical data. Data obtained included immunosuppression regimen, incidence and type of graftspecific complications, acute rejection, and graft failure. Acute rejection was defined as documented histological evidence of rejection or a reported increase in immunosuppression regimen due to decreased graft function clinically, with subsequent reversal of clinical dysfunction. Graft failure was defined as return to dialysis (renal failure), return to insulin usage for pancreas transplants, need for re-transplantation, or death.

\section{Statistical design}

Univariate statistics was performed to determine clinical differences between survey respondents and nonrespondents, and the effect of pregnancy on graft function. We subsequently used risk-adjusted stratified multivariable Cox proportional-hazards models with pregnancy as a time-varying covariate to estimate its effect of pregnancy on graft survival. We used age, race, and clinical covariates collected to risk adjust these estimates. The model structure was stratified by organ type to optimize any estimate of a pregnancy effect on long-term graft function. All statistical analyses were conducted using Stata 13 (College Station, TX, USA).

\section{Results}

Of the initial study cohort $(\mathrm{n}=532), 283$ subjects were reached and 152 patients responded to the survey ( $46 \%$ declined to participate). The overall survey response rate was $29 \%$ (64\% of reachable subjects in the study cohort). Eighty-two percent of respondents were kidney recipients, $19 \%$ were liver recipients, and $13 \%$ were pancreas recipients (Table 1). The demographics of the study population are displayed in Table 1. There were no significant differences at the $95 \%$ level between
Table 1. Frequency of demographics, graft complications, and adverse outcomes for survey respondents and nonrespondents

\begin{tabular}{|c|c|c|}
\hline Variable name & $\begin{array}{l}\text { Survey } \\
\text { respondents (\%) }\end{array}$ & Nonrespondents (\%) \\
\hline $\mathrm{n}$ & 152 & 529 \\
\hline Asian & $5(3.3)^{\star \star}$ & $6(1.1)^{\star \star}$ \\
\hline Black & $29(19.1)$ & 95 (18.0) \\
\hline Hispanic & $1(0.7)$ & $3(0.6)$ \\
\hline $\begin{array}{l}\text { American Indian/ } \\
\text { Alaska Native }\end{array}$ & $0(0)^{\star \star}$ & $3(0.6)^{\star \star}$ \\
\hline $\begin{array}{l}\text { Native Hawaiian/ } \\
\text { Pacific Islander }\end{array}$ & $0(0)^{\star *}$ & $3(0.4)^{\star \star}$ \\
\hline White & $113(74.3)$ & $379(75.1)$ \\
\hline Other & $2(2.6)$ & $11(2.1)$ \\
\hline Age $<35$ yr & $68(44.7)^{\star *}$ & $198(37.4)^{\star *}$ \\
\hline Age $>35 y r$ & $84(55.3)^{\star *}$ & $331(62.6)^{\star \star}$ \\
\hline \multicolumn{3}{|l|}{ Type of organ } \\
\hline Kidney & $125(82.24)$ & $422(79.8)$ \\
\hline Liver & $29(19.1)$ & $107(20.2)$ \\
\hline Pancreas & 20 (13.2) & $58(11.0)$ \\
\hline Death & $0(0)$ & $118(22.3)$ \\
\hline Lifetime rejection & $11(7.24 \%)$ & $29(5.5)$ \\
\hline Lifetime graft loss & $24(15.8)^{*}$ & $187(35.4)^{\star}$ \\
\hline Lifetime complication & $52(34.2)^{*}$ & $133(25.1)^{*}$ \\
\hline \multicolumn{3}{|l|}{ Type of complication } \\
\hline Infection & $27(17.8)$ & $73(13.8)$ \\
\hline Reoperation & $14(9.2)$ & $39(7.4)$ \\
\hline Acute post-op & $13(8.6)$ & $46(8.7)$ \\
\hline $\begin{array}{l}\text { Renal failure requiring } \\
\text { dialysis }\end{array}$ & $9(5.9)^{\star *}$ & $17(3.2)^{\star \star}$ \\
\hline Diabetes mellitus & $6(4.0)$ & $13(2.5)$ \\
\hline Delayed graft function & $2(1.3)^{\star \star}$ & $16(3.0)^{\star \star}$ \\
\hline
\end{tabular}

*Statistically significant difference at the $p<0.05$ level.

** Statistically significant difference at the $p<0.10$ level.

survey responders and non-responders for race, age, type of organ transplanted, lifetime rejection, or specific types of complications; however, there were significant differences between responders and non-responders for lifetime graft loss and lifetime complications with respondents being less likely to have graft loss and more likely to have complications $(\mathrm{p}=0.037$ and $\mathrm{p}=0.036$, respectively).

\section{Prenatal counseling}

Fig. 2 illustrates survey responses regarding how patients were counseled about pregnancy after transplantation. One-third of survey respondents $(33.5 \%)$ were counseled against pregnancy by one or more providers (including obstetricians, hepatologists, nephrologists, and transplant specialists) at any point in time. Forty-four percent of all patients who became pregnant were counseled against pregnancy at some point after their transplantation, yet, still chose to become pregnant. 


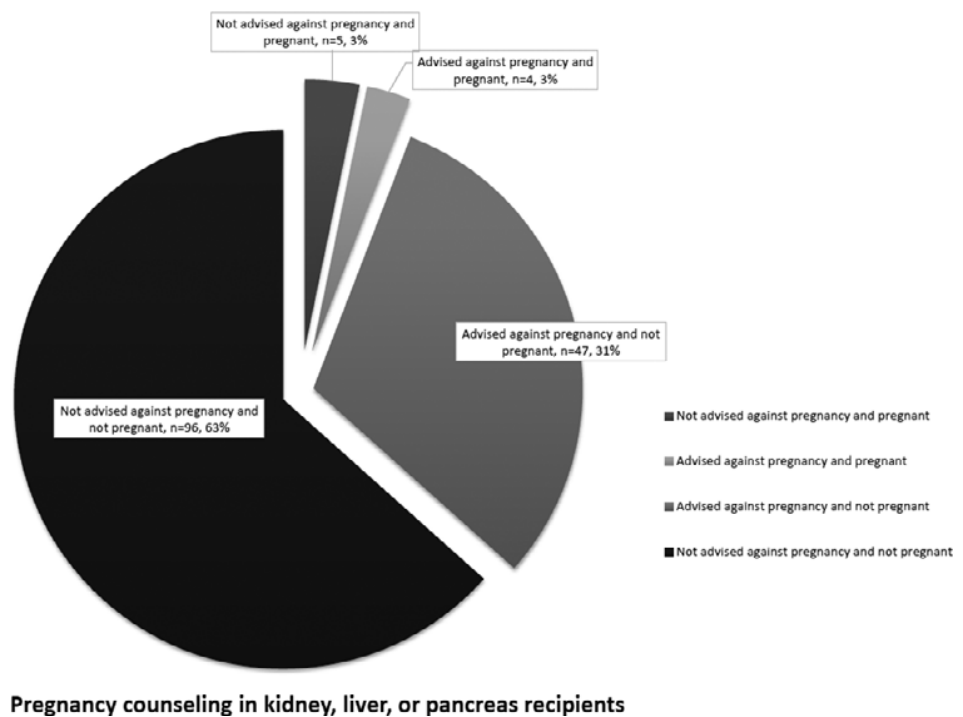

Fig. 2. Pregnancy counseling in kidney, liver, or pancreas recipients. Survey responses surrounding pregnancy counseling during the transplant process and eventual pregnancy status after transplant. The majority of patients were not counseled against becoming pregnant and never became pregnant, but a third of patients were advised to never become pregnant and never did.

Pregnancy, delivery, and neonatal complications

Nine patients $(5.9 \%)$ who responded to the survey were able to become pregnant, which resulted in a total of 17 pregnancies because some subjects had multiple pregnancies (Table 2). The 17 pregnancies resulted in eight live births $(47 \%)$,

Table 2. Demographics, graft complications, and adverse outcomes for pregnant and nonpregnant survey respondents

\begin{tabular}{llc}
\hline Variable name & $\begin{array}{l}\text { Pregnant } \\
\text { subjects (\%) }\end{array}$ & $\begin{array}{c}\text { Nonpregnant } \\
\text { subjects (\%) }\end{array}$ \\
\hline $\mathrm{n}$ & 9 & 143 \\
Asian & $0(0)^{\star}$ & $5(3.5)^{\star}$ \\
Black & $1(11.1)$ & $28(19.6)$ \\
Hispanic & $0(0)$ & $1(0.7)$ \\
White & $8(88.9)$ & $105(73.4)$ \\
Age $<35$ yr & $7(77.8)^{\star}$ & $61(42.7)^{\star}$ \\
Age $>35$ yr & $2(22.2)^{\star}$ & $82(57.3)^{\star}$ \\
Type of organ & & \\
Kidney & $6(66.7)$ & $119(83.2)$ \\
Liver & $3(33.3)$ & $26(18.8)$ \\
Pancreas & $1(11.1)$ & $19(13.3)$ \\
Pregnancy prior to Txp & $0(0)$ & $87(60.8)$ \\
Lifetime rejection & $3(33.3)^{\star *}$ & $8(5.6)^{\star \star}$ \\
Lifetime graft loss & $2(22.2)^{\star}$ & $22(15.4)^{\star}$ \\
Lifetime complication & $9(100)^{\star}$ & $43(30.1)^{\star}$ \\
Type of complication & & \\
Infection & $5(55.6)^{\star}$ & $22(15.4)^{\star}$ \\
Reoperation & $3(33.3)^{\star *}$ & $11(7.7)^{\star *}$ \\
Acute post-op & $5(55.6)^{\star}$ & $8(5.6)^{\star}$ \\
Renal failure requiring dialysis & $4(44.4)^{\star}$ & $5(3.5)^{\star}$ \\
Diabetes mellitus & $1(11.1)$ & $5(3.5)$ \\
Delayed graft function & $0(0)$ & $2(1.4)$ \\
\hline
\end{tabular}

*Statistically significant difference at the $p<0.05$ level.

${ }^{*}$ Statistically significant difference at the $p<0.10$ level. eight early embryonic demises $(47 \%)$, and one stillbirth $(5.9 \%)$. Seven of 12 women actively attempting conception were unsuccessful, resulting in a $58.3 \%$ infertility rate among our respondents. Complications were present in $88 \%$ of women with pregnancies resulting in a live birth, including gestational diabetes, hypertension, and preeclampsia, with $50 \%$ of subjects requiring additional antenatal medical therapies including antihypertensives to manage blood pressure and diabetic medications to reduce hyperglycemia (Table 3). Of the eight live births, $50 \%$ were born prematurely, and with a median birth weight of $2.9 \mathrm{~kg}$. Twelve percent of women reported neonatal complications including jaundice. Twenty-five percent of children were diagnosed with chronic medical complications, including asthma and developmental delay.

\section{Graft-related complications}

Univariate analysis demonstrated women with pregnancies post-transplantation trended toward higher rates of acute rejection than nulliparous recipients, although this difference was not statistically significant (pregnant vs. nulliparous, 33\% vs. $5.6 \%, \mathrm{p}=0.07)$. Furthermore, when parous vs. nulliparous patients were stratified by organ transplant type, there were no statistically significant differences in acute rejection ( $\mathrm{p}=0.12$ for kidney, $\mathrm{p}=0.26$ for liver, and too small of a sample size for pancreas).

Graft survival for pregnant vs. nulliparous transplant recipients was statistically different at the 
Prenatal counseling, pregnancy, and transplant outcomes

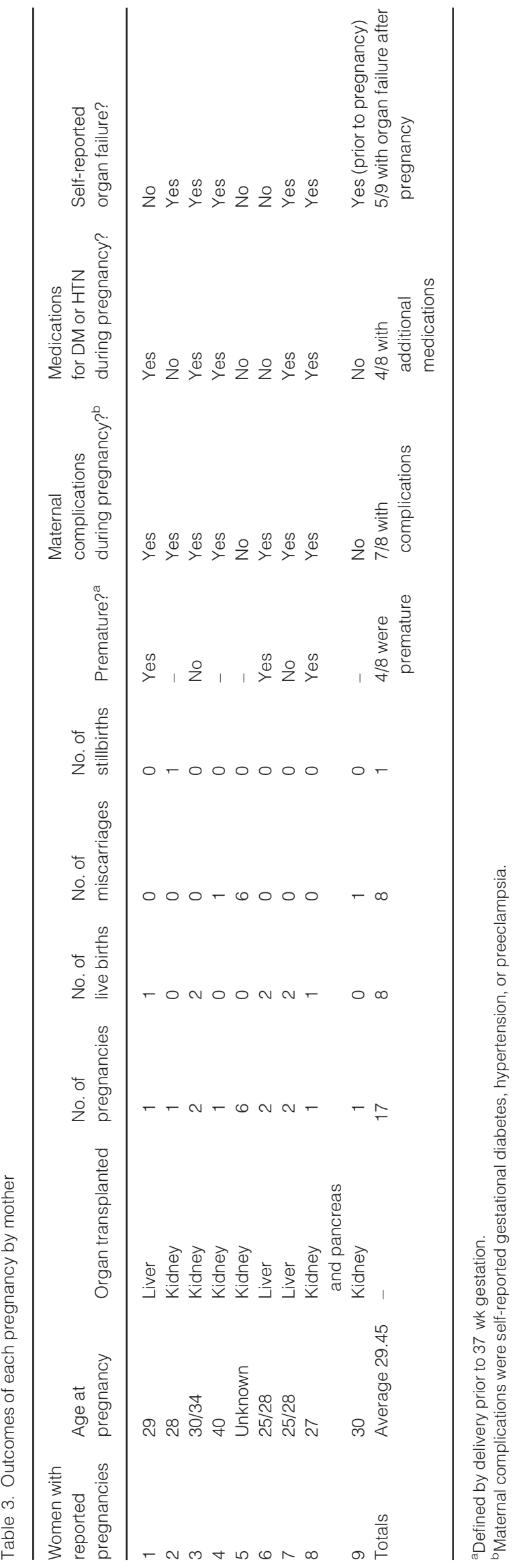

95\% level on univariate analysis; however, there were no significant differences in graft survival when the population was stratified by each organ subgroup. On multivariate analysis, pregnancy did not have a significant effect on graft survival after transplant $(\mathrm{HR}=1.00,95 \%$ CI $0.99-1.01)$ after stratifying for organ type and adjusting for demographics and clinical covariates (Fig. 3).

\section{Discussion}

The clinical benefit of abdominal organ transplantation in managing end-organ failure in young reproductive-aged women is undeniable. After transplantation, renal and endocrine functions rapidly return and fertility is often restored. Posttransplant survival has reached an all-time high, which has led to more focused attention on posttransplant quality of life (9-11). Among the most important of these concerns is the possibility of regaining fertility, becoming pregnant, having children, and the risks inherent to this pursuit for the woman and the fetus. In this study, we conducted a survey of female abdominal organ transplant recipients from a large academic transplant center to assess the nature of preconception counseling focused on pregnancy, delivery, and maternal and neonatal outcomes. We identified significant variation in how patients were being counseled, and observed cases of active dissuasion by transplant providers, despite the increased but acceptable risks and associated outcomes of pregnancy after transplant. Our findings illustrate that significant gaps exist in improving prenatal counseling by transplant and obstetric providers.

Although most pregnant solid organ recipients are managed as high-risk obstetrical patients, standard management procedures or protocols have yet to be implemented for managing this population on a wide scale. Despite consensus from the American Society of Transplantation that pregnancy is usually safe one yr post-transplantation given that certain criteria are met, most providers have continued to recommend that patients wait two yr before becoming pregnant $(1,12)$. The factors to be achieved prior to pregnancy include: stable allograft function without episodes of rejection occurring in the past year, stable immunosuppressive regimens consisting of agents without known teratogenic risk, and low-risk status for harmful opportunistic infections $(13,14)$. Further studies are needed to better characterize outcomes and risks of pregnancy in solid organ recipients. Studies demonstrate that the majority of transplant providers currently advise women considering preg- 


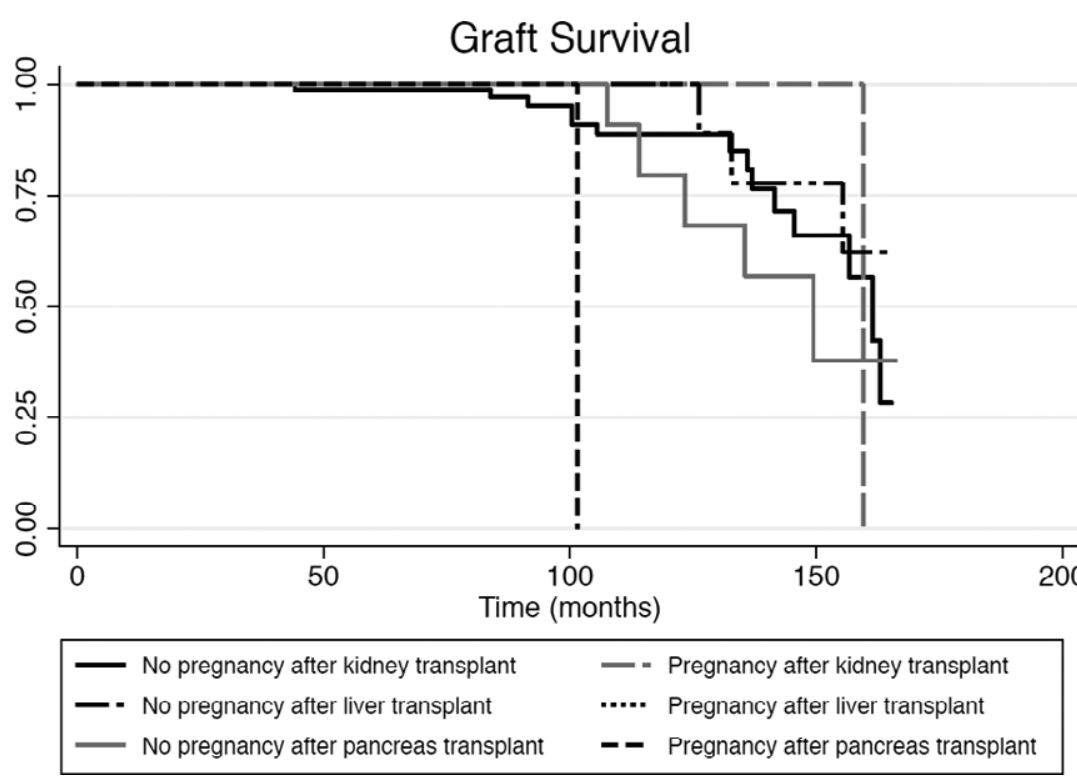

Fig. 3. Kaplan-Meier graft survival estimates of patients according to graft type and previous pregnancy status.

nancy based on "attending physician's discretion" as opposed to patient preference or protocols (8). Standardized management practices would ideally optimize outcomes for this unique population.

The principle aim of this analysis was to better understand how transplant recipients are counseled about the risks of pregnancy. In our study, $33.5 \%$ of respondents said they were advised against pregnancy by at least one provider, which included obstetricians, transplant surgeons, hepatologists, and nephrologists. This is similar to what is reported in a large Canadian study, and is concerning in the context of relatively benign risk-adjusted long-term graft outcomes (15). Furthermore, among patients who successfully conceived, 45\% were advised against pregnancy. This phenomenon calls for early counseling discussions with transplant recipients of reproductive age prior to conception to mitigate risks to both the patient and offspring in an effort to remedy this observed gap in provider understanding of the risks and current guidelines. Efforts to further educate providers on standard of care guidelines should be initiated to disseminate knowledge more broadly. Further study on factors associated with delayed discussions of fertility and pregnancy with patients should be undertaken (16).

The self-reported risk of complications in pregnancy found in our analysis is consistent with previous studies (17). Our data showed that the rate of obstetric complications was significantly higher at $100 \%$ of our pregnant liver transplant patients $(\mathrm{n}=3)$, and at $88 \%$ of our entire study cohort than the general population, consistent with previous studies; previously reported rates of obstetric complications in liver transplant recipients were over $50 \%$ (4). The literature shows that liver and kidney organ recipients have significantly higher rates of preeclampsia and gestational diabetes in comparison with the general US population $(4,5)$.

Furthermore, adverse delivery outcomes, including preterm delivery and low birth weight, are also well established in this population (18). The rate of premature delivery in our study cohort $50 \%$ of live births) was also significantly higher than is present in the general population $(11 \%)$, according to the Centers for Disease Control (13). The transplant literature states that infants delivered to female transplant recipients have a 50\% likelihood of being born premature, and nearly a $20 \%$ risk of having intrauterine growth restriction $(3,19)$, well above the $12.5 \%$ rate of prematurity and $5 \%$ risk of intrauterine growth restriction in the general US population $(14,20,21)$. The high incidence of prematurity has been associated with developmental delays, although this risk has not been well characterized due to the limited longitudinal data on offspring outcomes (12). The implications of these increased risks are clinically important to the care and counseling of patients as they assess the risks and benefits of pregnancy after transplant. These results support that transplant patients who become pregnant need to be carefully monitored throughout pregnancy, ideally with a high-risk obstetrician, in an effort to mitigate poor outcomes from potential pregnancy complications (16). These expanded efforts and improved partnering 
Prenatal counseling, pregnancy, and transplant outcomes

between patients, obstetricians, and transplant providers may improve maternal, fetal, and neonatal outcomes in this growing at risk population (16).

Unfortunately, we were unable to examine the clinical outcomes of the children born from parents with solid organ transplants. The reported incidence of chronic medical conditions in these children was interesting, but requires more detailed analysis. Within the general population, the rate of asthma is $9 \%$ (22) and developmental delay is $13.9 \%$ (23), which are similar to the rate found in our study. Further research, from multiple centers, may shed additional light on the specific risks to the children of transplant recipients and affect how neonatal counseling should be directed for this patient cohort.

Despite the poignant findings regarding how transplant patients are counseled, our study had numerous limitations. The survey design exposes our conclusions to risks of recall bias. More complete information on specific complications would further sharpen our understanding of risks associated with pregnancy in this population. Additionally, our study was limited by its observational nature and cohort selection. Future studies should examine outcomes in patients who underwent transplantation prior to age of 18 , a cohort which was excluded and for whom risks associated with pregnancy are especially relevant. Understanding how women are counseled regarding the period of time to avoid pregnancy post-transplantation would be beneficial. A follow-up study of providers' counseling practices at this center might not only illuminate the current practices of providers and explain reasoning for recommendations including clinical factors such as immunosuppressive regimen and stability of graft but also could shed light on the effectiveness of providers' discussions with patients. Additionally, such a study could demonstrate how provider practices have changed since American Society of Transplant Surgeons (ASTS) pregnancy guidelines were released in 2005. These details could guide creation of more robust guidelines for transplant and obstetric providers.

Pregnancy after transplant is an important clinical issue that warrants further study to better inform patients and providers about the risks and optimal management considering this important life decision. Educating physicians and patients about the risks associated with pregnancy after transplantation is crucial to improve patient care. Further studies with pooled data from multiple centers are warranted to better counsel patients and providers.

\section{References}

1. McKay DB, Josephison MA. Pregnancy in recipients of solid organs-effects on mother and child. N Engl J Med 2006: 354: 1281.

2. Armenti VT. National Transplant Registry. Philadelphia, PA: Thomas Jefferson University, 2012.

3. Armenti VT, Radomski JS, Moritz MJ, Gaughan WJ, McGrory CH, Coscia LA. Report from the National Transplantation Pregnancy Registry (NTPR): outcomes of pregnancy after transplantation. Clin Transplant 2003: 20: 131.

4. Deshrande NA, James NT, Kucirka LM et al. Pregnancy outcomes of liver transplant recipients: a systematic review and meta-analysis. Liver Transpl 2012: 18: 621.

5. Deshrande NA, James NT, Kucirka LM et al. Pregnancy outcomes in kidney transplant recipients: a systematic review and meta-analysis. Am J Transplant 2011: 11: 2388.

6. Gutierrez MJ, Acebedo-Ribo M, Garcia-Donaire JA et al. Pregnancy in renal transplant recipients. Transplant Proc 2005: 37: 3721

7. Galdo T, Gonzalez F, Espinoza M et al. Impact of pregnancy on the function of transplanted kidneys. Transplant Proc 2005: 37: 1577.

8. McKay DB, Adams PL, Bumgardner GL et al. Reproduction and pregnancy in transplant recipients: current practices. Prog Transplant 2006: 16: 127.

9. Matas AJ, Smith JM, Skeans MA et al. OPTN/SRTR 2012 Annual Data Report: kidney. Am J Transplant 2014: 14(Suppl 1): 11.

10. Israni AK, Skeans MA, Gustafson SK et al. OPTN/ SRTR 2012 Annual Data Report: pancreas. Am J Transplant 2014: 14(Suppl 1): 45.

11. Kim WR, Smith JM, Skeans MA et al. OPTN/SRTR 2012 Annual Data Report: liver. Am J Transplant 2014: 14(Suppl 1): 69.

12. Stratta P, Canavese C, Giacchino F, Mesiano P, QuaGLiA M, Rossetti M. Pregnancy in kidney transplantation: satisfactory outcomes and harsh realities. J Nephrol 2003: 16: 792 .

13. Centers for Disease Control and Prevention. Available from: http://www.cdc.gov/reproductivehealth/maternalinfanthealth/pretermbirth.htm. [cited 10/30/2013], 2013.

14. Romo A, Carceller R, Tobajas J. Intrauterine growth retardation (IUGR): epidemiology and etiology. Pediatr Endocrinol Rev 2009: 6(Suppl 3): 332.

15. Humphreys RA, Wong HH, Milner R, Matsuda-AbediNI M. Pregnancy outcomes among solid organ transplant recipients in British Columbia. J Obstet Gynaecol Can 2012: 34: 416.

16. McKay DB, Josephison MA, Armenti VT et al. Reproduction and transplantation: report on the AST Consensus Conference on Reproductive Issues and Transplantation. Am J Transplant 2005: 5: 1592.

17. Blume C, Sensoy A, Gross MM et al. A comparison of the outcome of pregnancies after liver and kidney transplantation. Transplantation 2013: 95: 222.

18. Bouattar T, Hakim H, Rhou H, Benamar L, Bayahia R, Ouzeddoun N. Pregnancy in renal transplant recipients. Transplant Proc 2009: 41: 1586.

19. Davison JM, Bailey DJ. Pregnancy following renal transplantation. J Obstet Gynaecol Res 2003: 29: 227.

20. Goldenberg RL, Culhane JF. Low birth weight in the United States. Am J Clin Nutr 2007: 85: 584S.

21. Martin JA, Hamilton BE, Ventura SJ, Osterman MJK, Mathews TJ. Births: final data for 2011. Natl Vital Stat Rep 2013: 62: 1. 


\section{Rupley et al.}

22. Centers for Disease Control and Prevention. Available from: http://www.cdc.gov/asthma/asthmadata.htm. [cited 2/16/2014], 2014.
23. Centers for Disease Control and Prevention. Available from: http://www.cdc.gov/features/dsdev_disabilities/ [cited 2/16/2014], 2014.

\section{Appendix 1. Patient Phone Survey}

Patient Phone Survey

1. Name:

2. Date of birth:

3. What is the location where your transplant was performed?

4. Where do you currently receive medical care regarding your transplant?

5. Have you ever become pregnant prior to transplant? $(\mathrm{Y} / \mathrm{N})$

6.1. Did you use any contraceptives following your transplant? $(\mathrm{Y} / \mathrm{N})$

6.2. What type of contraceptive? (Abstinence, barrier, OCP/Nuvaring/patch, IUD, Implanon)

6.3. How long did you use contraceptives for? (months)

7. Did you have a hysterectomy, tubal ligation, or other permanent sterilization procedure prior to your transplant? (Y/N)

8. Did you develop diabetes requiring medication post-transplant prior to any pregnancy? (Y/N)

9. Did you develop hypertension or high blood pressure requiring medication post-transplant prior to any pregnancy? (Y/N)

10.1. Did you ever become pregnant after your transplant (repeat per pregnancy- record sequentially oldest to youngest)? (Y/N)

10.2. If so, how many pregnancies?

10.3. Was the pregnancy planned? $(\mathrm{Y} / \mathrm{N})$

10.4. What was the length of the pregnancy? (number)

10.5. What was the delivery/termination date? (weeks)

10.6. What hospital did you go to the for delivery/termination? (name, city, state)

10.7. Did you have a miscarriage? $(\mathrm{Y} / \mathrm{N})$

10.8. Did you have a termination? $(\mathrm{Y} / \mathrm{N})$

10.9. Did you have any complications during your pregnancy? $(\mathrm{Y} / \mathrm{N})$

10.10. Did you take any medication for hypertension or high blood pressure during your pregnancy? $(\mathrm{Y} / \mathrm{N})$

10.11. Did you take any medication for diabetes during your pregnancy? (Y/N)

11. This section is regarding the outcome of any pregnancies you had.

11.1. What was the baby's sex? (M/F)

11.2. What was the baby's birth weight? (lbs, oz)

11.3. Was the baby healthy? ( $\mathrm{Y} / \mathrm{N})$

11.4. What was the baby's Apgar scores? (number, number, number)

11.5. Was the baby premature $(\mathrm{Y} / \mathrm{N})$ ?

11.6. Did the baby have complications? $(\mathrm{Y} / \mathrm{N})$

11.7. Was the baby stillborn? (Y/N)

11.8. How old is the child now? (age in years)

11.9. Does the child have any chronic medical conditions? (Y/N)

11.10. What are they? (specify conditions)

12.1. This section is on how your organ functioned before, during, and after pregnancy. Has your organ ever failed? $(\mathrm{Y} / \mathrm{N})$

12.2. If so, when?________ (date)

12.3. Did your organ fail prior to pregnancy? $(\mathrm{Y} / \mathrm{N})$

12.4. Did your organ fail during pregnancy? $(\mathrm{Y} / \mathrm{N})$

12.5. Did your organ fail following delivery? $(\mathrm{Y} / \mathrm{N})$

13. Immunosuppressant regimen at time of pregnancy:

13.1. Were you taking Neoral (cyclosporine capsules and oral solution)? (Y/N)

13.2. Were you taking Sandimmune (cyclosporine)? ( $\mathrm{Y} / \mathrm{N})$

13.3. Were you taking other cyclosporine formulations? ( $\mathrm{Y} / \mathrm{N})$

13.4. Were you taking Prograf (tacrolimus capsules and injection, or FJ506)? (Y/N)

13.5. Were you taking Cellcept (mycophenolate mofetil capsules)? (Y/N)

13.6. Were you taking Imuran (azathioprine)? (Y/N)

13.7. Were you taking Rapamune (sirolimus)? (Y/N)

13.8. Were you taking Myfortic (mycophenolic acid)? ( $\mathrm{Y} / \mathrm{N})$

13.9. Were you taking steroids (prednisone)? (Y/N)

13.10. Were you taking other immunosuppressants? (Y/N) (Specify)

13.11. Did you have any changes to immunosuppressant regimen during pregnancy? (Y/N)

(Specify)

14. Did you ever breastfeed following organ transplant? ( $\mathrm{Y} / \mathrm{N})$

14.1. For how long? ___ (length in weeks)

14.2. What was your immunosuppressant regimen while breast feeding? (Specify) 
Prenatal counseling, pregnancy, and transplant outcomes

15. Were you actively trying to become pregnant since your transplant?

16. Did any of your physicians tell you not to get pregnant? If so, which type of physician told you this?

Would you like to receive follow-up information regarding the results of the study?

If so, what is your address?

This concludes the survey. Thank you for your time. 